\title{
Simulation of Hall Thruster Plumes in a Vacuum Chamber Using a Hybrid Method
}

\author{
Tyler D. Huismann ${ }^{1}$ and Iain D. Boyd ${ }^{2}$ \\ University of Michigan, Ann Arbor, MI 48109
}

\begin{abstract}
A hybrid particle-fluid method is applied to model the plume from a Hall thruster operated in the $12 \mathrm{~V}$ vacuum chamber at the Arnold Engineering Development Center. The approach utilizes the direct simulation Monte Carlo method and the Particle in Cell method to simulate xenon neutrals and xenon ions. A fluid electron model is utilized that is based on the Boltzmann relation. The complex geometry of the facility is modeled using an unstructured mesh. Specific facility operation priorities are addressed such as evaluation of thermal loads to the baffles in the vacuum chamber. Code accuracy is assessed through comparison of simulation results with experimental data taken in the plume of a 20kW class Hall thruster. Reasonable agreement is shown between the experimental and simulation results although a strong sensitivity to assumed thruster exit conditions is demonstrated.
\end{abstract}

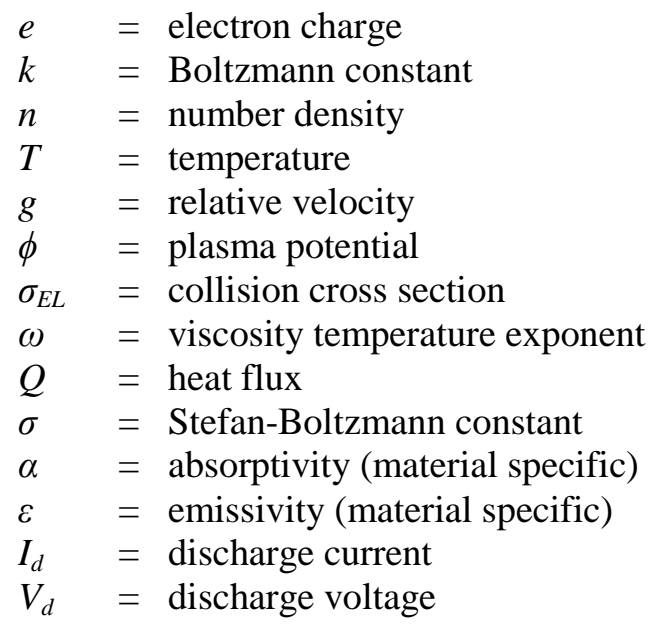

\section{Nomenclature}

I. Introduction

$\mathrm{H}$ ALL thrusters are an efficient propulsion option for spacecraft, with high specific impulses and low thrust levels making them particularly well suited for station-keeping maneuvers. However, a primary concern regarding the use of Hall thrusters is the effect of their plumes. Possible negative effects include spacecraft contamination, material erosion, and communications interference, all of which emphasize the importance of plume modeling with regards to spacecraft integration. Hall thruster plume modeling has been reviewed by Boyd ${ }^{1}$ where it was determined that hybrid fluid-particle methods are the most successful. An important aspect of plume modeling involves assessment using ground-based experiments conducted in vacuum chambers. One of these types of

\footnotetext{
${ }^{1}$ Graduate Student, Department of Aerospace Engineering, AIAA Student Member.

${ }^{2}$ Professor, Department of Aerospace Engineering, AIAA Associate Fellow. 
facilities is the Arnold Engineering Development Center (AEDC) 12' diameter, vertically oriented vacuum chamber $(12 \mathrm{~V})$. This facility provides both experimental and computational analysis for customers, and it is the focus of the present work to continue development of simulation tools that can be applied at AEDC specifically for the analysis of Hall thruster plumes in $12 \mathrm{~V}$.

In general, the plume of a Hall thruster consists of neutrals, energetic ions, and electrons. Plume behavior is complicated by the multiple types of collisions involved and different physical phenomena, for example: collisions due to thermal velocity, collisions between neutrals and ions involving charge exchange, and self-consistent electric fields. Furthermore, in ground-based facilities the presence of background gases must be accounted for. Computational methods are uniquely suited for this task since physical models allow for varying degrees of fidelity. In this paper, a hybrid fluid-particle simulation method is used to investigate a Hall thruster plume in the $12 \mathrm{~V}$ vacuum chamber, extending previous work done on the subject ${ }^{2}$. Specifically, the direct simulation Monte Carlo (DSMC) method $^{3}$ is used to incorporate collision dynamics, and the particle-in-cell (PIC) method ${ }^{4}$ is used to capture electric field effects. The complex geometry of the chamber is handled using an axisymmetric unstructured mesh. Additional capabilities specific to the needs of operation in $12 \mathrm{~V}$ are discussed. Some of the primary needs are solution quality assessment and computation of the thermal loads to the facility. The facility uses a set of baffles to direct the plume to cryo-pumps at the bottom of the chamber (see Figure 1). Since the plume impinges directly on these surfaces, the associated thermal loads are a concern.



Figure 1: Mesh for the 12V Chamber

In this paper, several steps taken to support these needs are outlined below. Details of the numerical method are outlined in Section II, including a sensitivity study performed to evaluate the effects of key input parameters on the simulations. The results of the simulation begin in Section III, including field data, thermal loads computation, and comparison with experiment. Following the implementation of the thermal loads module, some of the underlying physics of this implementation are explored. Finally, Section IV contains concluding remarks. 


\section{Numerical Simulation}

\section{A. Collision Dynamics}

The numerical simulations use a hybrid fluid-particle method to model the plume. A DSMC module handles collisions between heavier particles $\left(\mathrm{Xe}, \mathrm{Xe}^{+}\right.$, and $\left.\mathrm{Xe}^{++}\right)$, such as neutral-neutral and ion-neutral collisions. The DSMC method uses virtual particles to simulate collisions in rarefied gas flows. The particles represent real ions and neutrals and are grouped in cells whose characteristic lengths are shorter than a mean free path. Pairs of these particles are selected at random and a collision probability is evaluated that is proportional to the product of the relative velocity and collision cross-section. This probability is compared to a random number to determine if the collision occurs. If so, collision dynamics are performed to alter the colliding particles properties.

Two types of collision dynamics are relevant to Hall thruster plumes: elastic (momentum exchange) collisions and charge exchange collisions. Elastic collisions involve only exchange of momentum between participating particles. The Hall thruster plume is confined to two different types of momentum exchange collisions, neutralneutral collisions and neutral-ion collisions. For neutral-neutral collisions, the variable hard sphere model is employed $^{3}$. The cross-section for xenon is:

$$
\sigma_{E L}(X e, X e)=\frac{2.12 \times 10^{-18}}{g^{2 \omega}} m^{2} \quad \text { and } \quad \omega=0.12
$$

where $g$ is the relative velocity and $\omega$ is related to the viscosity temperature exponent for xenon. For neutral-ion elastic interactions, the cross-sections measured by Miller et al..$^{5}$ are used:

$$
\begin{gathered}
\sigma_{E L}\left(X e, X e^{+}\right)=\left(175.26-27.2 \log _{10}(g)\right) \times 10^{-20} \mathrm{~m}^{2} \\
\sigma_{E L}\left(X e, X e^{++}\right)=\left(103.26-17.8 \log _{10}(g)\right) \times 10^{-20} \mathrm{~m}^{2}
\end{gathered}
$$

Isotropic scattering is assumed for both types of elastic collisions. Charge exchange collisions pertain to the transfer of one or more electrons between an atom and an ion. The cross sections are assumed to follow the same expressions for neutral-ion elastic collisions. However, it is also assumed there is no transfer of momentum accompanying the charge exchange, since it is primarily a long-range interaction.

\section{B. Plasma Dynamics}

The PIC module is used to move the heavier ion particles that are influenced by the electric fields present, whereas the lighter electrons are modeled as a fluid. The PIC module determines the charge density at the nodes in the mesh based on the proximity of each particle to the surrounding nodes. The charge density is then used to compute the potential at each node. This is accomplished by incorporating the Boltzmann relation for the electrons. The Boltzmann relation uses several assumptions applied to the electron momentum equation, such as the fluid electron flow being collisionless, isothermal, no magnetic fields present, and that the electron pressure obeys the ideal gas law, to arrive at the following ${ }^{8}$ :

$$
\phi=\phi_{r e f}+\frac{k T_{r e f}}{e} \ln \left(\frac{n_{e}}{n_{r e f}}\right)
$$

where $\phi$ is the plasma potential, $\phi_{r e f}$ is a reference potential, $k$ is Boltzmann's constant, $e$ is the electron charge, $T_{r e f}$ the constant electron reference temperature, and $n_{e}$ and $n_{r e f}$ the local electron number density and reference electron number density, respectively. Reference values are taken at the thruster exit plane and the parameters used in this study can be found in Table 1. The assumption of quasi-neutrality is employed to obtain the electron number density from the ion number densities. The potential is then differentiated spatially to obtain the electric fields that transport the ions. 


\section{Boundary Conditions}

For computations of a Hall thruster plume in $12 \mathrm{~V}$, boundary conditions must be specified at the thruster exit and along all solid surfaces in the computational domain, including cryo-pumps and baffles. Some of the macroscopic properties of the plume are required at the thruster exit, namely the plasma potential, electron temperature, and number density, velocity, and temperature of each heavy species in the calculation. Since the particles exit the thruster with an unknown radial velocity component, this results in a velocity vector that is not parallel to the centerline of the chamber. The angle between the center-line and velocity vector is referred to as the divergence angle, and since it is not known a priori, a sensitivity study is performed that includes a range of assumed divergence angles. Particles that exit the thruster plane are assigned a radial velocity component that varies linearly with the distance from the center of the thruster channel. Therefore, a particle exiting the center of the channel will have no radial velocity component, while a particle exiting the channel near its inner or outer wall will have the highest radial velocity component and the angle of the velocity vector with respect to the center-line for that particle will be the divergence angle specified. The ion temperature at the thruster exit is also unknown and so results are generated for two different values.

The other thruster exit macroscopic properties are determined in general via a combination of analysis and intelligent estimation ${ }^{5}$. See Table 1 for the listing of experimental operating conditions and Table 2 for the listing of thruster exit simulation parameters used to replicate those operating conditions. It should be noted that the BHT20K has undergone almost no detailed characterization and so the determination of appropriate thruster exit conditions is much more difficult than for the Aerojet BPT-4000 thruster studied in $12 \mathrm{~V}$ previously ${ }^{2}$.

Concerning the different types of surfaces in $12 \mathrm{~V}$, each one is assumed to have a plasma potential of zero, including the baffles, which are electrically grounded. The floors and outer walls of the chamber are liquid nitrogen $\left(\mathrm{LN}_{2}\right)$ cooled and thus are set to the cryogenic $\mathrm{LN}_{2}$ temperature of $77 \mathrm{~K}$. The cryo-pumps located at the bottom of the chamber are cooled with gaseous helium $(\mathrm{GHe})$ and are set to the cryogenic temperature of $20 \mathrm{~K}$. All ions that collide with any wall are neutralized. When a particle strikes the cryo-pump surfaces, a fraction of those particles are pumped away. This process is characterized by a sticking coefficient (a value of 0.8 is used for the present study). For the particles scattered back into the flow field from all surfaces, diffuse reflection is assumed which is characterized by the surface temperature.

The boundary conditions for the baffles are different than other solid surfaces in that they incorporate a variable surface temperature. Their outer surfaces are exposed to the $\mathrm{LN}_{2}$ panels so their temperature is set initially to $77 \mathrm{~K}$. As the simulation progresses, heat fluxes to the baffles are known and the wall temperature can be updated. This allows for the tracking of the time history of the thermal load to the baffles as discussed in the next section.

\begin{tabular}{|c|c|c|c|c|c|c|}
\hline Condition & $\dot{\boldsymbol{m}}$ & $\boldsymbol{I}_{\boldsymbol{d}}$ & $\boldsymbol{V}_{\boldsymbol{d}}$ & $\boldsymbol{\phi}_{\boldsymbol{r e f}}$ & $\boldsymbol{n}_{\text {ref }}$ & $\boldsymbol{T}_{\boldsymbol{r e f}}$ \\
\hline Point 1 & $19.53 \mathrm{mg} / \mathrm{s}$ & $21.2 \mathrm{~A}$ & $500 \mathrm{~V}$ & $50 \mathrm{~V}$ & $1.77 \times 10^{17} 1 / \mathrm{m}^{3}$ & $2 \mathrm{eV}$ \\
\hline Point 2 & $58.60 \mathrm{mg} / \mathrm{s}$ & $100.0 \mathrm{~A}$ & $200 \mathrm{~V}$ & $20 \mathrm{~V}$ & $1.57 \times 10^{18} 1 / \mathrm{m}^{3}$ & $2 \mathrm{eV}$ \\
\hline
\end{tabular}

Table 1: Operating conditions and simulation parameters

\begin{tabular}{|c|c|c|c|c|c|c|}
\hline \multirow{2}{*}{ Species } & \multicolumn{2}{|c|}{ Number Density $\left(\mathbf{1} / \mathbf{m}^{\mathbf{3}}\right)$} & \multicolumn{2}{c|}{ Axial Velocity $(\mathbf{m} / \mathbf{s})$} & \multicolumn{2}{c|}{ Temperature $(\mathbf{K})$} \\
\cline { 2 - 7 } & Point 1 & Point 2 & Point 1 & Point 2 & Point 1 & Point 2 \\
\hline $\mathrm{Xe}$ & $1.28 \times 10^{18}$ & $1.06 \times 10^{19}$ & 281.3 & 274.9 & 750 & 750 \\
\hline $\mathrm{Xe}^{+}$ & $1.18 \times 10^{17}$ & $1.31 \times 10^{18}$ & 27792.0 & 14518.0 & 23188 & 23188 \\
\hline $\mathrm{Xe}^{++}$ & $2.96 \times 10^{16}$ & $1.30 \times 10^{17}$ & 39304.0 & 20531.0 & 23188 & 23188 \\
\hline
\end{tabular}

Table 2: Specific simulation input parameters at the thruster exit 


\section{Thermal Loads}

A new model for determining the heat flux to the baffle walls of the facility is introduced, which couples the convective heat flux to the wall at each time step to the wall temperature. These baffles are designed to direct the plume in $12 \mathrm{~V}$ into a lower chamber where cryogenic pumps are located. The sides of the baffles exposed to the plume are covered in aluminum foil $\left(\varepsilon_{\mathrm{Al}}=0.6\right)$ whereas the sides exposed to the chamber walls are fabricated from stainless steel $\left(\varepsilon_{S S}=0.54, \alpha_{S S}=0.2\right)$. Whenever a particle impinges on one of the baffles, the incident and reflected properties determine the convective heat flux to the wall. The following equilibrium expression is used to compute the wall temperature based on the heat flux imparted to it:

$$
Q+\sigma\left(\alpha_{s s} T_{c}^{4}-\varepsilon_{s S} T_{b}^{4}-\varepsilon_{A l} T_{b}^{4}\right)=0
$$

where $Q$ is the convective heat flux imparted to the wall, $\sigma$ is the Stefan-Boltzmann constant, $\varepsilon$ is the emissivity of the material (with $S S$ denoting stainless steel and $A l$ denoting aluminum), $\alpha$ is the absorptivity of the material, $T_{c}$ is the LN2 cryogenic temperature, and $T_{b}$ is the baffle temperature we wish to compute.

The thermal loads imparted to each baffle are a function of the operating point of the thruster in the chamber, as well as the divergence angle of the thruster. Therefore a sensitivity study is performed to compare loads at a high specific impulse configuration (Point 1) and a high thrust configuration (Point 2) through a range of assumed divergence angles.

The baffles under consideration are impinged upon by an ionized gas. Since the baffles in the facility are electrically grounded, there is a resulting potential difference, $\Delta \phi$, between the plume and the baffle surface. This results in a "sheath" around the surface ${ }^{7,8,9}$ in which the positively charged ions are accelerated towards the surface. Since most of the heat flux is due to the ions, a basic energy balance model is incorporated to quantify this effect. The heat flux to each surface is directly proportional to the kinetic energy of the particle that impinges upon it. Thus, once the potential around a surface is computed, the acceleration due to the sheath is evaluated as follows:

$$
\frac{1}{2} m_{\text {ion }} v_{\text {ion }}^{2}+C \Delta \phi=\frac{1}{2} m_{\text {ion }} v_{\text {ion, baffle }}^{2}
$$

where $C=e$ for $\mathrm{Xe}^{+}$ions and $C=2 e$ for $\mathrm{Xe}^{++}$ions. The overall effect expected of this model is an increase in heat flux, since the ions impinge upon the baffles at a greater velocity due to the sheath.

\section{Plume Simulation Results}

The DSMC simulations presented use a total of 300,000 simulated particles at steady state over a domain of 1853 triangular cells. The simulation runs for 800,000 time-steps to reach a steady state and then for another 200,000 time-steps to sample macroscopic data. The time-step size is $2 \times 10^{-6}$ seconds, resulting in a total simulation time of 2 seconds.

\section{A. Sensitivity to Operating Conditions}

The results shown below in Figures 2 and 3 correspond to field data for operating condition 1, whereas Figures 5 and 6 correspond to operating condition 2. The heat flux shown in Figures 2 and 5 is evaluated approximately as:

$$
Q=\frac{1}{2} \rho u^{3}
$$

Figures 4 and 7 correspond to the predicted thermal loads for operating points 1 and 2, respectively. It can be seen in Figures 2 and 5 that the heat flux is focused like a beam past Baffles 1 and 2. However, Baffle 3 reduces the area through with the plume can flow, increasing the number of particles impinging on the surface which in turn increases the heat transfer to Baffle 3. The plasma potential in Figures 3 and 6 is well-behaved, which is expected when the Boltzmann relation is used. 


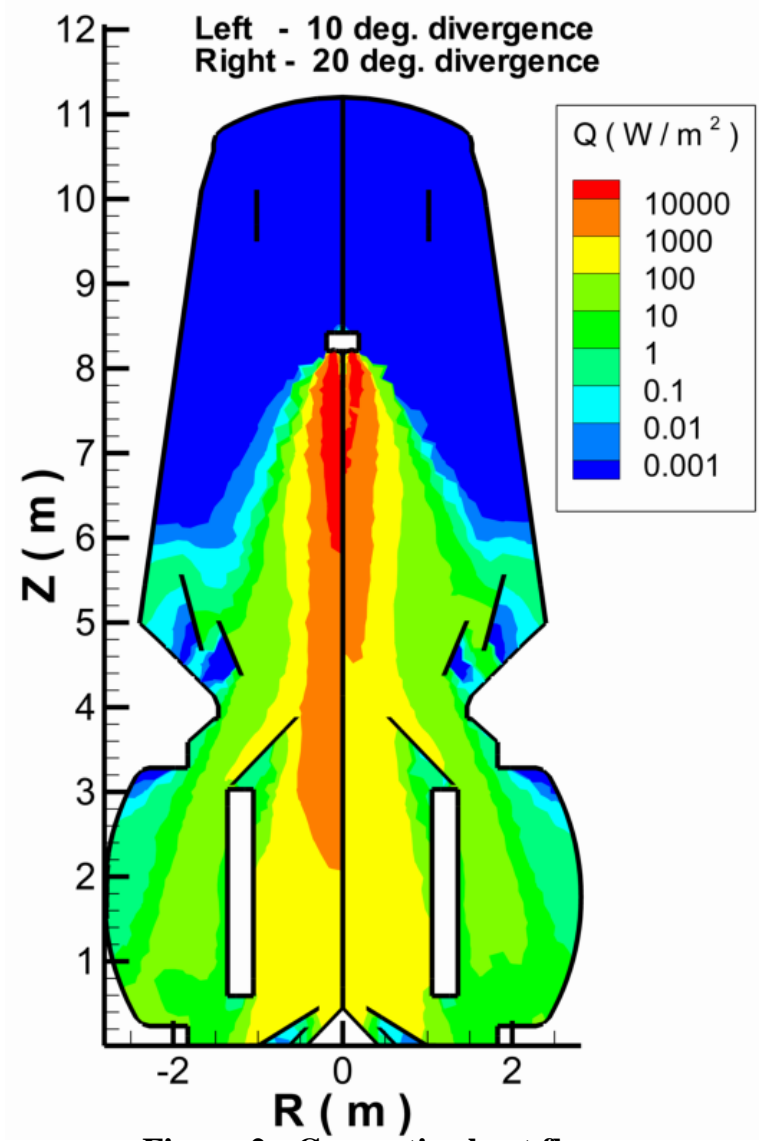

Figure 2: Convective heat flux, Operating Point 1

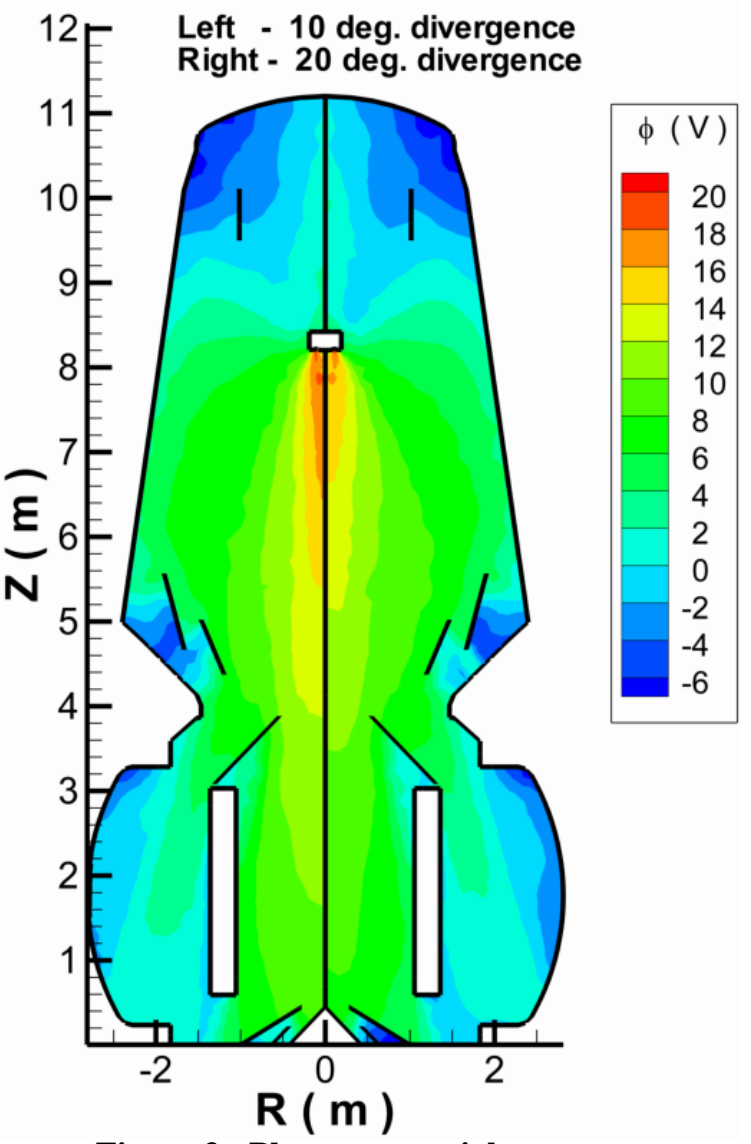

Figure 3: Plasma potential, Operating Point 1



Figure 4: Thermal loads, Operating Point 1 


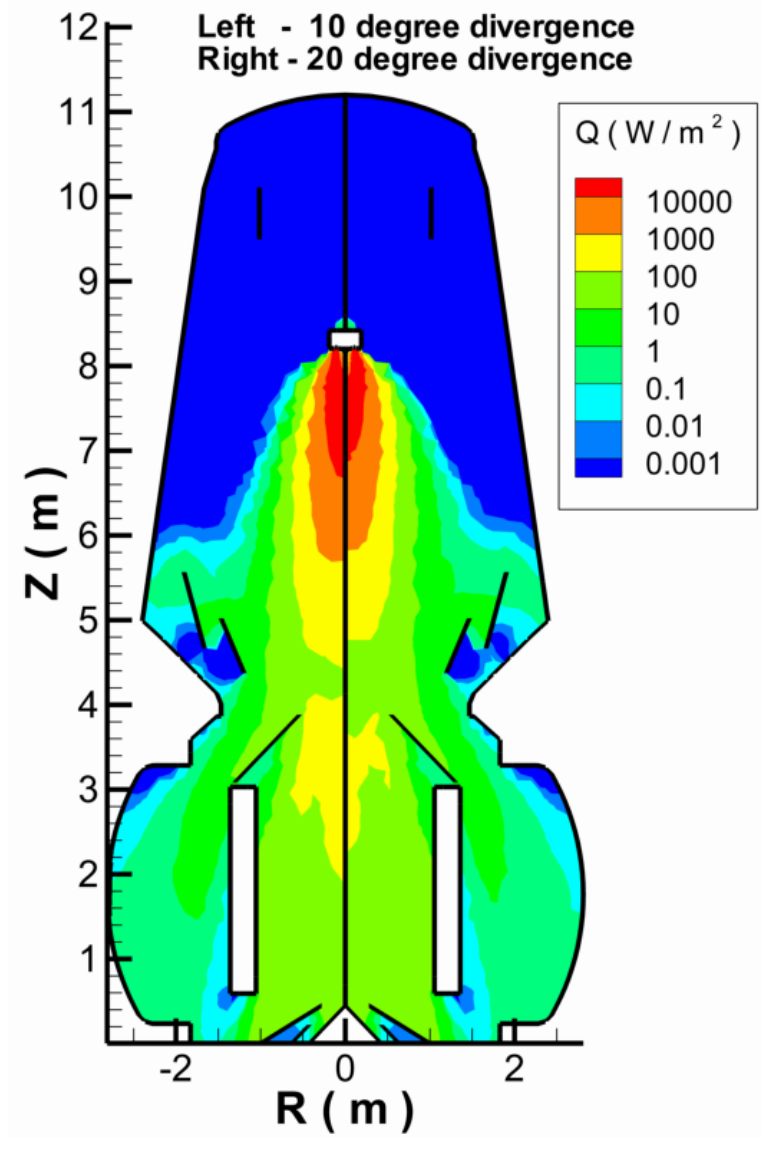

Figure 5: Convective heat flux, Operating Point 2

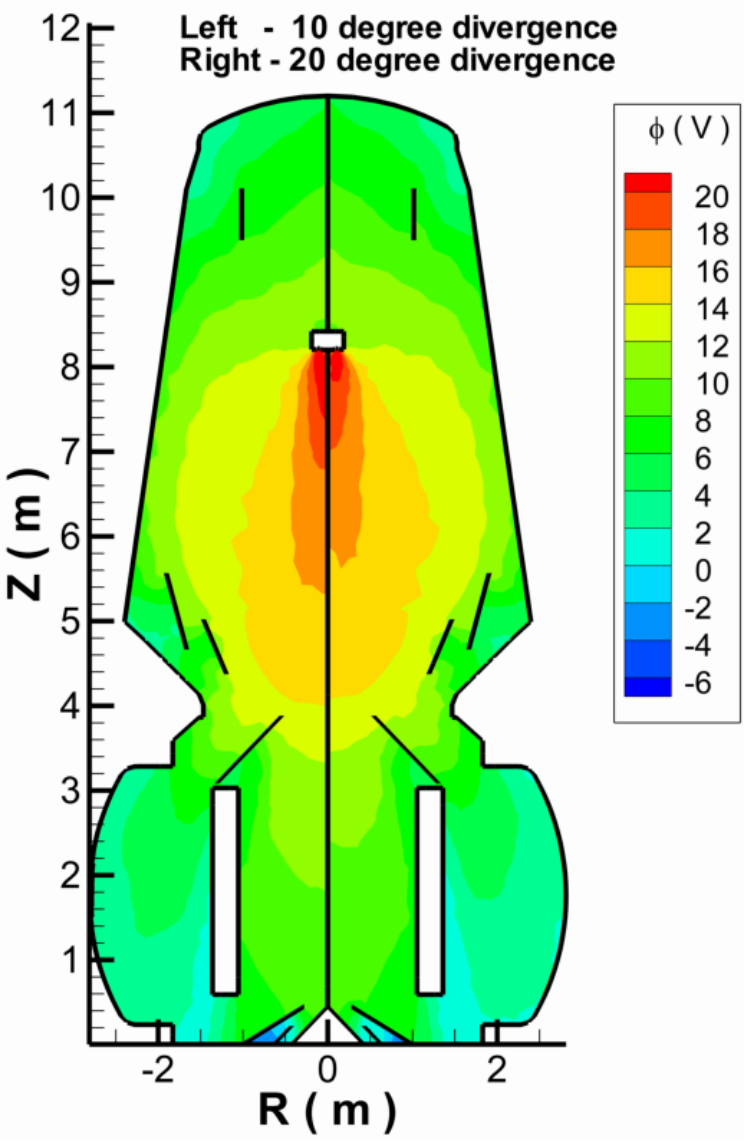

Figure 6: Plasma potential, Operating Point 2

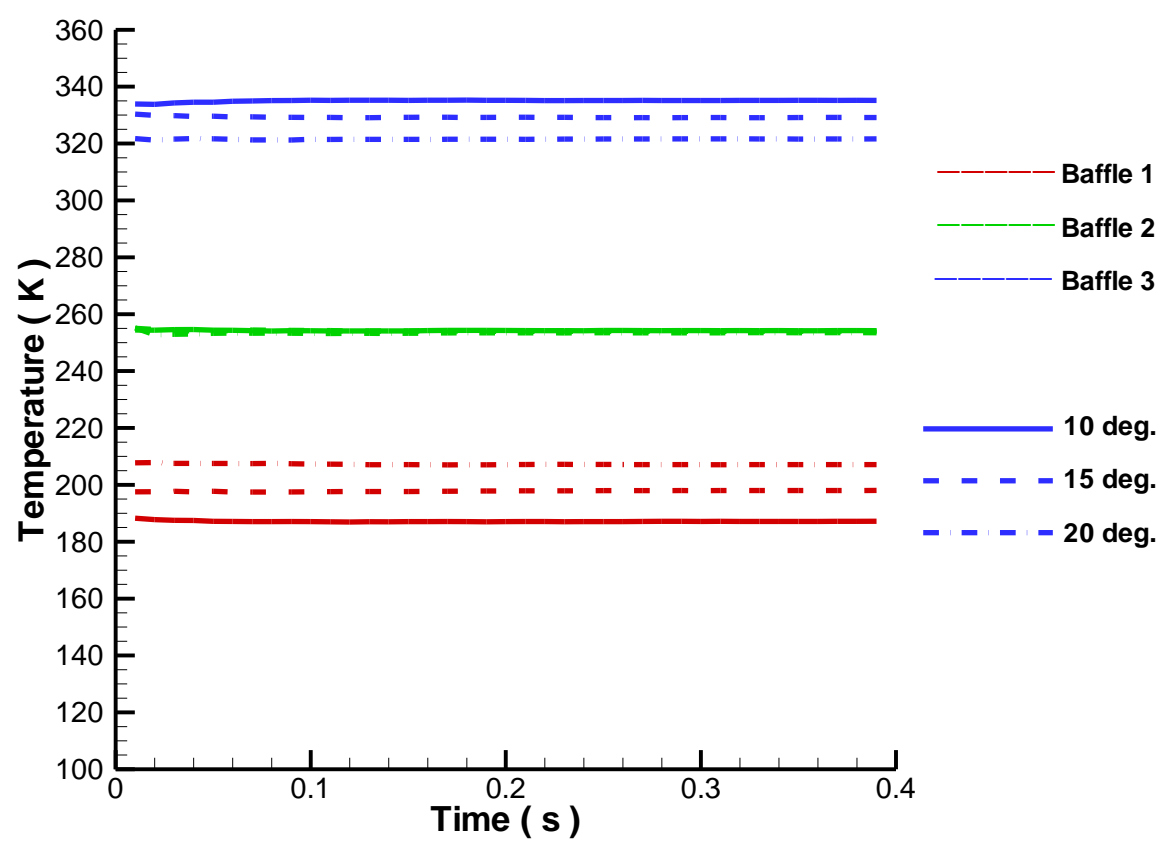

Figure 7: Thermal loads, Operating Point 2 
The data for Baffles 1, 2, and 3 correspond to the 12V schematic shown in Figure 1. The differences in baffle temperature between the two operating points are clear from Figures 4 and 7, as well as the effects of the assumed divergence angle. A smaller value of divergence angle results in a more beam-like plume, in which case the convective heat flux is focused more towards the centerline of the chamber. Since Baffle 3 is closest to the centerline, it makes physical sense that the smallest divergence angle results in its largest thermal load. Likewise, Baffles 1 and 2 respond with larger thermal loads at higher divergence angle. Baffle 2 in particular appears to experience a maximum load somewhere between $10^{\circ}$ and $20^{\circ}$ (apparent in Figure 4). While operating point 1 displays a higher convective heat flux, the total thermal load to the baffles is not much different from operating point 2. This is due to the fact that in a high $I_{s p}$ configuration, particles exiting the thruster are moving much faster than in a high thrust configuration, but less mass overall is being ejected. In general, operating point 1 exhibits a higher sensitivity to choice of divergence angle than operating point 2 .

Results are also obtained utilizing the sheath model described in Section II-D, and comparisons are shown below in Figures 8 and 9 assuming a $10^{\circ}$ divergence angle.

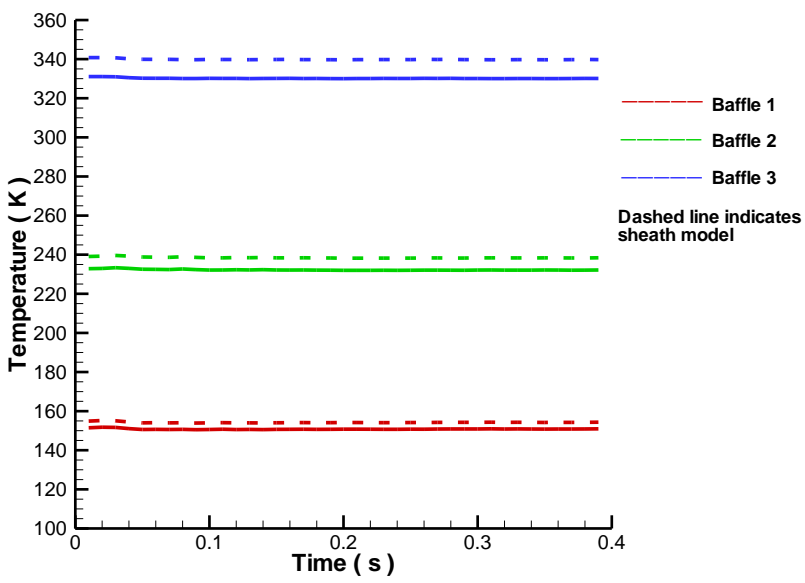

Figure 8: Thermal loads with sheath model, Operating Point 1

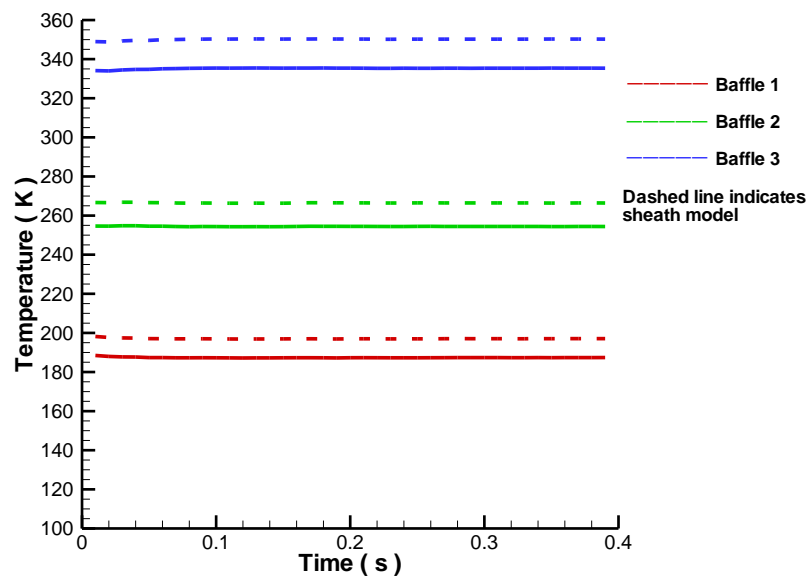

Figure 9: Thermal loads with sheath model, Operating Point 2

As can be seen, the sheath model results in about a 3-4\% increase in baffle temperature, corresponding to a 12$17 \%$ increase in heat flux.

\section{B. Comparisons to Experiment}

Measured data is reported in Reference 6 corresponding to the experiments performed in $12 \mathrm{~V}$ for the Busek BHT-20K Hall thruster at the operating conditions under consideration here. Data specific to the baffle thermal loads are shown in Table 3 below. Model assessment is also performed through comparison with data measured by a set of Faraday cup probes whose locations are shown in Figure 1. Data specific to ion current densities and Faraday cup probes can be found in Table 4 and Figures 10 and 11.

The discrepancies with experimental data concerning baffle thermal loads range from roughly $0.5-1.5 \%$ without the sheath model and about 2-5\% with the model. Comparison of predicted temperatures with the hot and cold side of the baffles results in an error magnitude range of roughly 4\%-7\% without the sheath model and 1\%-9\% with the sheath model in place. In terms of heat fluxes, the associated errors lie in the range of $2-40 \%$. 


\begin{tabular}{|c|c|c|c|c|c|c|c|}
\hline Condition & $\begin{array}{c}\text { Baffle 3 } \\
\text { North }\end{array}$ & $\begin{array}{c}\text { Baffle 3 } \\
\text { South }\end{array}$ & $\begin{array}{c}\text { Average, } \\
\text { N and S }\end{array}$ & $\begin{array}{c}\text { Baffle 3, } \\
\text { Predicted, } \\
\text { No sheath }\end{array}$ & $\begin{array}{c}\text { Baffle 3, } \\
\text { Predicted, } \\
\text { With sheath }\end{array}$ & $\begin{array}{c}\text { \% Difference, } \\
\text { No Sheath }\end{array}$ & $\begin{array}{c}\text { \% Difference, } \\
\text { With Sheath }\end{array}$ \\
\hline Point 1 & $316.0 \mathrm{~K}$ & $351.5 \mathrm{~K}$ & $333.7 \mathrm{~K}$ & $328.3 \mathrm{~K}$ & $339.8 \mathrm{~K}$ & $-1.62 \%$ & $1.83 \%$ \\
\hline Point 2 & $320.5 \mathrm{~K}$ & $346.7 \mathrm{~K}$ & $333.6 \mathrm{~K}$ & $335.2 \mathrm{~K}$ & $350.3 \mathrm{~K}$ & $0.47 \%$ & $4.99 \%$ \\
\hline
\end{tabular}

Table 3: Baffle 3 temperatures: comparison of measurement and simulation

\begin{tabular}{|c|c|c|c|c|c|}
\hline & $\begin{array}{c}\text { Measured } \\
\text { Current Density }\end{array}$ & $\begin{array}{c}\text { Predicted Current } \\
\text { Density } \\
\left(\mathbf{1 0}^{\circ} \text { divergence }\right)\end{array}$ & $\begin{array}{c}\text { Predicted Current } \\
\text { Density } \\
(\mathbf{2 0} \text { divergence })\end{array}$ & $\begin{array}{c}\text { \%Difference, } \\
\mathbf{1 0}^{\circ} \text { divergence }\end{array}$ & $\begin{array}{c}\text { \% Difference, } \\
\mathbf{2 0}^{\circ} \text { divergence }\end{array}$ \\
\hline Point 1 & $0.0348 \frac{\boldsymbol{A}}{\mathbf{m}^{\mathbf{2}}}$ & $0.0512 \frac{\boldsymbol{A}}{\mathbf{m}^{\mathbf{2}}}$ & $0.0478 \frac{\boldsymbol{A}}{\mathbf{m}^{\mathbf{2}}}$ & $47.1 \%$ & $37.2 \%$ \\
\hline Point 2 & $0.466 \frac{\boldsymbol{A}}{\mathbf{m}^{\mathbf{2}}}$ & $0.691 \frac{\boldsymbol{A}}{\mathbf{m}^{\mathbf{2}}}$ & $0.641 \frac{\boldsymbol{A}}{\boldsymbol{m}^{\mathbf{2}}}$ & $48.3 \%$ & $37.4 \%$ \\
\hline $\begin{array}{c}\text { Ratio of } \\
\text { Points 1 and 2 }\end{array}$ & 13.37 & 13.50 & 13.39 & $0.97 \%$ & $0.15 \%$ \\
\hline
\end{tabular}

Table 4: Comparison with measured Faraday cup data

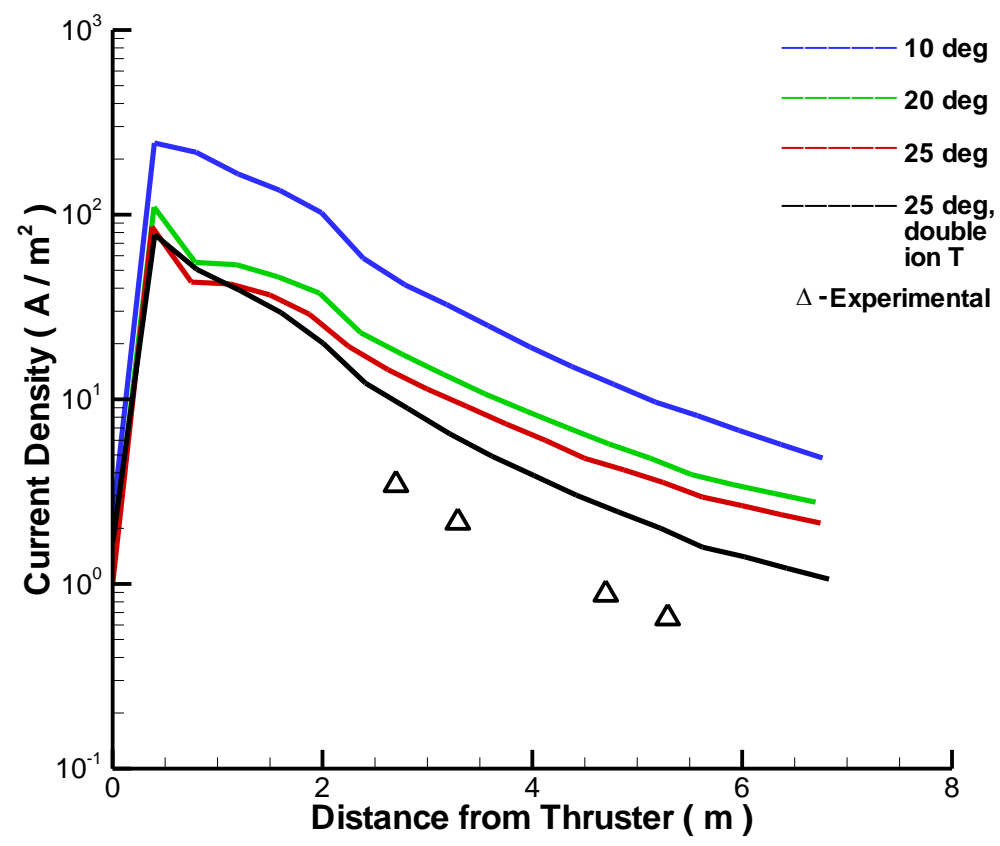

Figure 10: Profiles of ion current density along the plume axis, Operating Point 1 


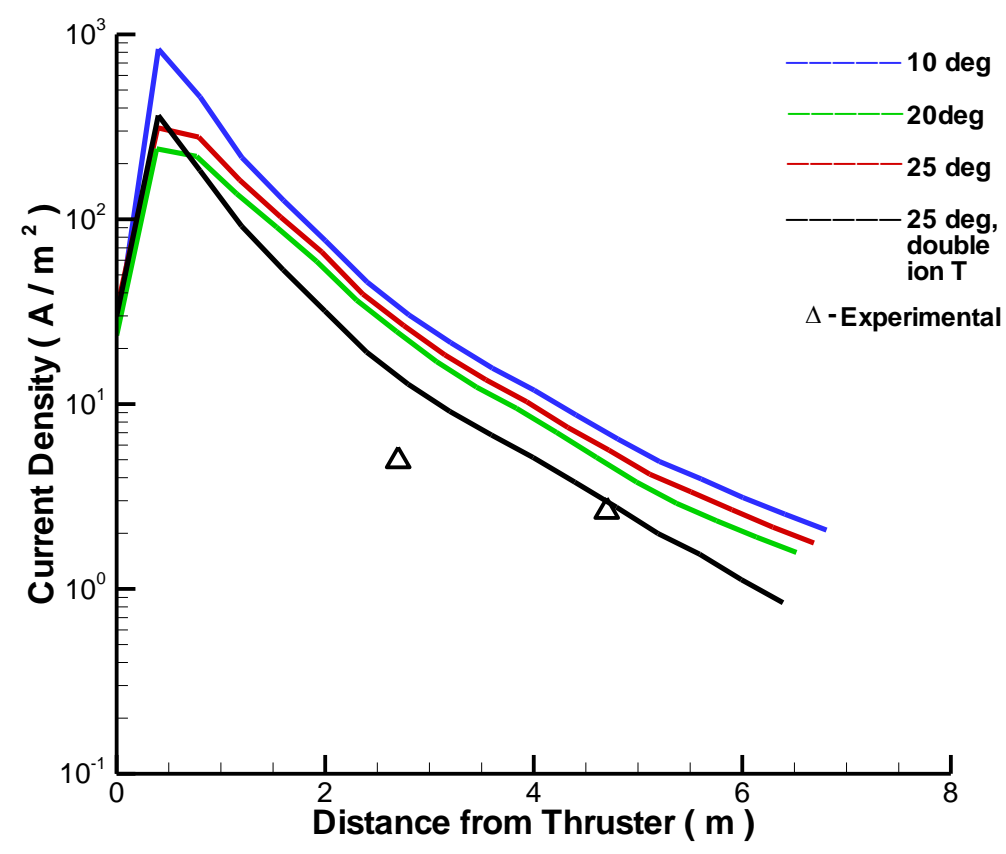

Figure 11: Profiles of ion current density along the plume axis, Operating Point 2

When comparing the baffle temperatures at operating points 1 and 2 with the measured data, there is excellent agreement with the measured data allowing for confident prediction of thermal loads for future tests. There is, however, a discrepancy between the North and South sides of the baffles. This is due primarily to two effects: uncertainties in plasma flow conditions at the thruster exit and supplemental cooling provided to the North side of the baffle during the test in $12 \mathrm{~V}$. Regarding the first point, while certain experimental quantities are known, the plasma flow conditions at the exit were not measured, requiring some of the boundary conditions there to be estimated. With regards to the second point, pre-experiment modeling suggested that Baffle 3 would experience the highest thermal loads. Therefore the North side of Baffle 3 was heat-sunk to the LN2 cooled panels located on the walls of the chamber with multiple braided copper straps. Since the simulations are performed on an axisymmetric unstructured domain, three-dimensional effects such as this cannot be considered.

The current density measurements were all taken with Faraday cup probes. The comparisons in Table 4 correspond with the probe at a $+28 \mathrm{~V}$ potential bias and located at the same axial position as the thruster exit plane and at a radial position of $0.54 \mathrm{~m}$ from the center-line of the chamber. The comparisons shown in Figures 10 and 11 correspond to a series of probes located along the center-line of the chamber at distances measured down-stream of the thruster exit.

The comparisons with experimental ion current density measurements at the thruster exit do not appear as promising as the predictions associated with the baffle thermal loads. However, the heat flux scales with $T_{b}^{4}$, thus when considering the magnitude of the error between prediction and experiment regarding heat flux, instead of an error of a factor of 1.005-1.05 an error of a factor of 1.02-1.2 is seen. Referring to Table 4, the error of ion current prediction at this location is roughly $40 \%$. This can be attributed to the two effects. The location of the probe exhibits extreme sensitivity in simulations, as a difference as little as $1-2 \mathrm{~cm}$ shows variation in current density by a factor of 5. The other contributing factor is the substantial positive voltage bias applied to the probe. This produces a potential hill that reduces the number of ions that reach the probe, an effect not captured in the simulations. Thus it is expected to compute higher values of current density than shown in the experimental data. However, the ratio of current density between the two operating points is closer to the measured ratio, signifying that effects between the operating conditions are captured in the simulations.

As can be seen in Figure 10 and 11, there appears to be general correlation with current density measured along the plume axis, but also a substantial over-prediction. The sensitivity analysis that was performed confirms that doubling the ion temperature increases the divergence of the plume, which reduces current density along the center- 
line of the chamber. However, this effect does not bring the simulation current density values much closer to the experimental data. This leads us to the conclusion that the discrepancy is due to the plasma dynamics model. Utilizing the Boltzmann relation imposes a smaller variation in plasma potential than seen in the real system. This leads to an under-prediction of ion acceleration, which in turn leads to a higher ion current density along the centerline.

\section{Conclusions}

A general purpose, hybrid DSMC-PIC code has been progressed from a simulation tool for plasma plumes in the $12 \mathrm{~V}$ electric propulsion facility to a tool that allows for the confident prediction of thermal loads. The level of physical modeling fidelity has been increased and the predictions correlate well with some of the measured data. It can be seen that the inclusion of the sheath model affects the predicted baffle temperatures, slightly increasing the thermal loads. Comparisons with measurements of ion current density show mixed results from excellent agreement to errors of $40 \%$. Consequently, further study is required to better understand the sensitivity of the computation to assumptions made for the thruster exit conditions as well as to determine more accurate plasma dynamics models.

\section{Acknowledgments}

The authors appreciatively acknowledge support for this work from the Air Force Office of Scientific Research through Grant FA9550-07-1-0123 as well as technical discussions with Ken Tatum and Kurt Polzin.

\section{References}

${ }^{1}$ Boyd, I.D., "Review of Hall Thruster Plume Modeling," Journal of Spacecraft and Rockets, Vol. 38, No. 3, 2001 , pp. $381-387$.

${ }^{2}$ Boyd, I.D., Sun, Q., Cai, C., and Tatum, K. E., "Particle Simulation of Hall Thruster Plumes in the 12V Vacuum Chamber," IEPC-2005-138, $29^{\text {th }}$ International Electric Propulsion Conference, October-November, 2005.

${ }^{3}$ Bird, G. A., Molecular Gas Dynamics and the Direct Simulation of Gas Flows, Clarendon Press, 1994.

${ }^{4}$ Birdsall, C. K., and Langdon, A. B., Plasma Physics via Computer Simulation, Adam Hilger, U.K., 1991.

${ }^{5}$ Miller, J. S., Pullins, S. H., Levandier, D. J., Chiu, Y., and Dressler, R. A., "Xenon Charge Exchange Cross Sections for Electrostatic Thruster Models," Journal of Applied Physics, Vol. 91, No. 3, 2002, pp. 984-991.

${ }^{6}$ Polzin, K.A., Szabo, J., Byrne, L., Raitses, Y., Dawbarn, A., Tatum, K.E., Crider, D., Brown, M., Bynum, K., Crews, D., Moeller, T., and Boyd, I.D., "Demonstration of High Power Plasma Propulsion Test Capabilities at Arnold Engineering Development Center" (ITAR Restricted)

${ }^{7}$ Bohm, D., The Characteristics of Electrical Discharges in Magnetic Fluids, McGraw-Hill, 1949.

${ }^{8}$ Chen, F.F., Introduction to Plasma Physics and Controlled Fusion, Vol. 1: Plasma Physics, Plenum Press, 1984.

${ }^{9}$ Lieberman, M.A., and Lichtenberg, A.J., Principles of Plasma Discharges and Materials Processing, Wiley-Intersience, 2005. 